\title{
Can 3D-CMR solve the apparent disassociation between carotid artery plaque and outcomes?
}

Robert W Biederman*1, Ronald B Williams ${ }^{1}$, Saundra B Gran ${ }^{1}$, June A Yamrozik ${ }^{1}$, Geetha Rayarao ${ }^{1}$, Vikas K Rathi ${ }^{1}$, Diane A Vido ${ }^{1}$, George Angheloiu², David R Neff ${ }^{3}$ and Mark Doyle ${ }^{1}$

Address: ${ }^{1}$ The Center for Cardiovascular Magnetic Resonance Imaging, The Gerald McGinnis Cardiovascular Institute at Allegheny General Hospital, Pittsburgh, PA, USA, ${ }^{2}$ DuBois Regional Medical Center, DuBois, PA, USA and ${ }^{3}$ Merck/Schering-Plough Pharmaceuticals, Inc., North Wales, PA, USA

* Corresponding author

from 13th Annual SCMR Scientific Sessions

Phoenix, AZ, USA. 21 -24 January 2010

Published: 21 January 2010

Journal of Cardiovascular Magnetic Resonance 2010, I2(SuppI I):PI40 doi:I0.I I86/I532-429X-I2-SI-PI 40

This abstract is available from: http://jcmr-online.com/content/I2/SI/PI 40

(c) 2010 Biederman et al; licensee BioMed Central Ltd.

Aggressive pharmacologic strategies dramatically lower incidence of MI and CVA. Yet, when examined at the arterial lumen, only nominal changes are seen. CMR, able to detect by 2D (single slice) underlying plaque characteristics, currently has not identified such features by 3D. A 3D volumetric approach may better define plaque 'vulnerability' while disentangling this conundrum.

\section{Hypothesis}

We hypothesize that in statin-naive pts with high grade carotid artery disease, 3D CMR integrates plaque components with lipid fractions superior to $2 \mathrm{D}$ while solving the 'disassociation' between outcome and \% stenosis.

\section{Methods}

Via CMR (1.5 T GE, WI), 860-two mm contiguous in vivo slices of advanced carotid disease $(>50 \%$; mean $63 \pm 22$ ) representing 38 complete bilateral human plaques (age $65 \pm 13$ yrs) were analyzed for 2D and 3D extent of vascular wall: lipid pool, fibrous cap, matrix and minima/ maxima of each. All were related to fasting lipids relative to \%stenosis via QPlaque (Medis, The Netherlands). Plaque morphology was determined by $\mathrm{T} 1$ and T2/PD.

\section{Results}

$35 / 38$ in vivo plaques were successfully imaged. Mean resolution: $1 \times 1 \times 2 \mathrm{~mm}$. The $\mathrm{mg} / \mathrm{dL}$ range of LDL was 63 -
186, HDL: 25-70 and TG: 81-213. Lipid pool represented $19 \pm 8 \%$ and fibrous plaque $9 \pm 24 \%$ of total vessel wall. $\mathrm{LDL}$, not total cholesterol $\left(\mathrm{Chol}_{\mathrm{T}}\right)$, was related to mean fibrous cap $(\mathrm{mm})(\mathrm{r}=0.6 \mathrm{p}=<0.05)$ while triglycerides were related to max fibrous cap but inversely to the lipid pool $(r=0.6,-0.5, p<0.05$ for both). The LDL:HDL and $\mathrm{Chol}_{\mathrm{T}}$ :HDL ratios were related to fibrous cap $(\mathrm{r}=0.6, \mathrm{p}<$ 0.05 for both). The $\mathrm{Chol}_{\mathrm{T}}$ : LDL was related to min fibrous cap $(\mathrm{mm})(\mathrm{r}=0.7 \mathrm{p}<0.05)$. Via 3D volumetrics only HDL was related to lipid volume while $\mathrm{Chol}_{\mathrm{T}}, \mathrm{LDL}, \mathrm{LDL}$ : HDL and $\mathrm{Chol}_{\mathrm{T}}: \mathrm{HDL}$ ratios were all related to fibrous cap volume ( $\mathrm{r}>0.5-0.7, \mathrm{p}<0.005$ for all). Importantly, relating $\%$ stenosis to any vessel wall component or its ratio revealed no relationship.

\section{Conclusion}

CMR can depict plaque composition demonstrating important relationships with common lipid fractions and even stronger relations via $3 \mathrm{D}$ volumetrics not visible by 2D approaches. Critically, in statin-naive pts, 3D CMR defined plaque morphology is highly related to 'clinical risk' not \% luminal stenosis, potentially serving as an easily identifiable marker and thus solving the quandary. 